Brief Report

\title{
Paeonol administration alleviates cognitive deficits and attenuates neural pathological changes in APP/PS1 mice
}

\author{
Shengxi Meng ${ }^{1, *}$, Bing Wang ${ }^{1}$, Wentao $\mathrm{Li}^{2}$ \\ ${ }^{1}$ Department of Traditional Chinese Medicine, Shanghai Jiao Tong University Affiliated Sixth People's Hospital, 200233 Shanghai, China \\ ${ }^{2}$ Department of Encephalopathy, Shanghai Municipal Hospital of Traditional Chinese Medicine, 200071 Shanghai, China \\ *Correspondence: mengshengxi@sjtu.edu.cn (Shengxi Meng) \\ DOI:10.31083/j.jin2004101 \\ This is an open access article under the CC BY 4.0 license (https://creativecommons.org/licenses/by/4.0/). \\ Submitted: 3 September 2021 Revised: 11 October 2021 Accepted: 20 October 2021 Published: 30 December 2021
}

\begin{abstract}
Alzheimer's disease typically presents with impaired cognition and pathological morphologic changes, including the accumulation of amyloid- $\beta$ plaques. Disease-modifying drugs are in urgent need as neuroprotective therapies. Exploration of novel therapeutics for alleviating symptoms of Alzheimer's disease has found promise in plant extracts of functional phenols. Paeonol is a water-soluble phenolic substance that has been shown to confer diverse biological effects, including neuroprotection. An Alzheimer's disease model of APP/PS1 double transgenic mice was used in this study, and the therapeutic effects of paeonol were assessed after three weeks' administration. It was found that paeonol treatment significantly increased behavioral performance in the Morris water maze test and increased discrimination rate in the novel object recognition test compared to vehicle-treated APP/PS1 mice. Histologically, paeonol treatment significantly alleviated the $A \beta$ plaque burden, reduced neural loss, inhibited microglia activation, and decreased neuroinflammation in the brain of APP/PS1 mice. In addition, a number of Alzheimer's disease-related synaptic plasticity deficits were ameliorated. The present results indicate that paeonol significantly relieved amyloid$\beta$ deposition and amyloid- $\beta$-mediated neuropathology in the brain of APP/PS1 mice, suggesting the potential of paeonol as a preventive and therapeutic agent for Alzheimer's disease.
\end{abstract}

Keywords

Paeonol; Alzheimer's disease; Cognitive deficits; Pathological changes; APP/PS1 mice

\section{Introduction}

Alzheimer's disease (AD) is one of the most common neurodegenerative disorders characterized by impairments of cognitive function [1]. The dominant pathological hallmarks of $\mathrm{AD}$ include aggregated amyloid- $\beta(\mathrm{A} \beta)$ peptides that form plaques, thus activating neuroinflammation and neuronal damage, which contribute to impaired cognitive function. Pathophysiological studies suggest that $\mathrm{A} \beta$ deposited throughout the brain plays a primary pathogenic role in $\mathrm{AD}$ [2]. Although our understanding of AD pathology has significantly advanced, we have not yet identified effective therapeutic drugs for the treatment of AD.
The amyloid- $\beta(\mathrm{A} \beta)$ hypothesis is widely recognized in $\mathrm{AD}$ research [3]. A number of studies suggest that $\mathrm{A} \beta$ deposited throughout the cortex and hippocampus contribute to the pathophysiology AD [4]. The APP/PS1 mouse is widely used in preclinical $\mathrm{AD}$ studies for its close connection with $\mathrm{A} \beta$ pathology. The mutations in the APP and PSEN1 genes induce $\mathrm{AD}$-like symptoms with a progressive $\mathrm{A} \beta$ deposition phenotype before aging $[5,6]$. The presence of $\mathrm{A} \beta$ peptides is also considered to have the secondary role of triggering microglial aggregation [7], with microglial activation amplifying neuroinflammation, producing reactive oxidative species and inflammatory cytokines (TNF- $\alpha$, IL$1 \beta$ ) [8], which contribute to furthering neurodegeneration in $\mathrm{AD}$. Though activated microglia have a beneficial role in $\mathrm{A} \beta$ clearance, chronic inflammation is detrimental and may lead to a dysregulated, irreversible inflammatory feedforward loop [9].

Paeonol is an herbal compound obtained from the Moutan cortex of Paeonia suffruticosa Andrews, found to possess various therapeutic pharmacological and biological properties [10]. A number of studies indicate that paeonol exerts multiple effects in the control of tissue homeostasis and the modulation of inflammatory mechanisms, with the potential to ameliorate neuronal damage [11-13]. This suggests a potential neuroprotective role of paeonol in AD. Though previous studies have explored the neuroprotective role of paeonol $[13,14]$, it remains unknown whether it could be beneficial in $\mathrm{AD}$. Hence, we performed an in vivo study of paeonol in a genetic rodent $\mathrm{AD}$ model. In order to reveal paeonol's therapeutic effect on $\mathrm{A} \beta$-related pathological changes and behavioral outcomes, we systemically administered paeonol into APP/PS1 transgenic mice that previously have been shown to robustly undergo $\mathrm{A} \beta$ plaque formation in the brain [15]. As such, our aim was to provide insights into the potential effect of paeonol as a novel therapeutic agent in AD. 


\section{Materials and methods}

\subsection{Animals}

APP/PS1 (APPswe, PSEN1dE9) double transgenic mice and their age matched wild-type (WT) littermates were purchased from Beijing Vital River Laboratory Animal Technology Co., Ltd. (Beijing, China). Mice were housed in specific pathogen free laboratory conditions with no more than four per cage. The mice were housed with controlled temperature $\left(22-24{ }^{\circ} \mathrm{C}\right)$ and humidity (55-65\%) under a $12 \mathrm{~h}$ darklight cycle, with food and water available ad libitum. All experiments were performed in accordance with The Guidelines for Animal Care and Use of China, and the experimental schemes were approved by the animal ethics committee of Shanghai Jiao Tong University, with every effect conducted to reduce animal suffering. After mice adapted to the experimenter and the environment, at the age of postnatal 8 months, we started the paeonol intervention study. Following the $3 \mathrm{R}$ principle (Replacement, Reduction, Refinement), we divided the mice into: vehicle-treated WT littermate control (WT), vehicle-treated APP/PS1 controls (APP/PS1) and paeonol-treated APP/PS1 mice (APP/PS1+ Pae). Each group contained 18 mice. Paeonol (Sigma-Aldrich, St. Louis, MO, USA, purity $\geq 99.5 \%$ ) was dissolved in carboxymethylcellulose sodium and was administered orally at a dosage of $20 \mathrm{mg} / \mathrm{kg}$ by an oral gavage for three consecutive weeks. The vehicle group was treated with the same dosage of carboxymethylcellulose sodium. Behavioral analyses were performed after a 3-week administration of paeonol. The dosage was determined by previous study [16] and our preliminary experimental results had confirmed that this dose had little effect on behavioral changes on WT mice.

\subsection{Morris water maze (MWM) test}

The MWM test is a hippocampus-dependent cognitive memory task assessed in an open field water maze as previously described [5]. The mice were trained to escape from the water by locating a hidden escape platform submerged approximately $1 \mathrm{~cm}$ below the water surface at a fixed location. The platform was located at the center of one of the four quadrants of the pool. Training consisted of 4 trials per day for 5 days. On day 6 of testing, the platform was removed and the mice were allowed to swim freely to explore the pool. The swimming parameters were recorded and calculated with a camera connected to a behavioral monitoring system (Noldus Information Technology, Wageningen, the Netherlands).

\subsection{Novel object recognition (NOR) test}

The object recognition test was performed in a square box under low light levels [17]. Mice were first habituated to the empty arena for $10 \mathrm{~min}$ per day for 3 days prior to the object recognition test. For the sampling phase, 2 identical objects were placed at 2 adjacent corners of the box, and the mice were allowed to explore freely in the box for $10 \mathrm{~min}$. After 24 $\mathrm{h}$, mice were reintroduced to the arena for $10 \mathrm{~min}$ for the test phase. During this test phase, the arena contained one object identical to those used in the sampling phase, and one novel object. The time spent exploring each object was recorded. A discrimination score was provided by calculating the time spent exploring the novel object minus the time spent exploring the familiar object, and dividing this by the total time spent exploring both novel and familiar objects during the test phase. The arena and objects were wiped down with 70\% ethanol between trials to minimize olfactory cues. Ethovision XT (Noldus, Wageningen, Netherlands) was used to track the mice during testing.

\subsection{Thioflavin S staining}

After the brain sections were permeabilized with $0.3 \%$ Triton X-100 and blocked in 5\% BSA, Thioflavin S (Thio S) staining was performed as previously described [18]. The hippocampal sections were immersed in Thio S solution (1\%, $8 \mathrm{~min}$ ) for staining. After washing, brain images were captured with a Nikon microscope. A $\beta$ plaque images were collected from six representative sections per mouse.

\subsection{ELISA}

After mice were deeply anesthetized with isoflurane, the cortex and hippocampus brains tissues were quickly dissected and homogenized. After the homogenate was centrifuged, the supernatants were collected and the samples used to measure TNF- $\alpha$, IL- $1 \beta, \mathrm{A} \beta 40$, and $\mathrm{A} \beta 42$ using commercial ELISA kits from Wako in accordance with the manufacturers' instructions.

\subsection{Stereotaxic injections}

Stereotaxic surgery and adeno-associated virus (AAV) injection were performed as previously described [19]. Mice were placed on a stereotaxic apparatus (RWD science, Shenzhen, China) under isoflurane anesthesia, and a solution containing AAV-GFP was delivered to CA1 region: $\mathrm{AP}-1.8 \mathrm{~mm}$; ML $1.25 \mathrm{~mm}$; DV $-1.25 \mathrm{~mm}$ in $100 \mathrm{~nL}$ doses. Infusions were performed at a rate of $20 \mathrm{~nL} / \mathrm{min}$. Spines of GFP-labeled neurons could be clearly visualized after a two-week recovery period.

\subsection{LTP}

Hippocampus tissue sections (400 $\mu$ m thick) were prepared with a Leica VT 1200 s sectioning system. A platinum stimulated electrode with a bipolar enamel coating was placed in the Schaeffer collateral pathway afferents under a microscope. The glass recording electrode was placed in the stratum radiatum of area CA1. 50\% of the maximum fEPSP was utilized to induce stable baseline synaptic transmission for 20-30 min prior to LTP induction. LTP was induced by three trains ( 5 min intertrain interval) of $100 \mathrm{~Hz}$ for $1 \mathrm{~s}$. Data were collected using CLAMPEX 9.0 (Axon Instruments, Burlingame, CA, USA) and presented as the average slope of the fEPSP. Clampfit 9.0 (Axon Instruments, Burlingame, CA, USA) was used to analyze data. Two-way ANOVA was used for electrophysiological data analysis with $p<0.05$ as the significance threshold. 
A

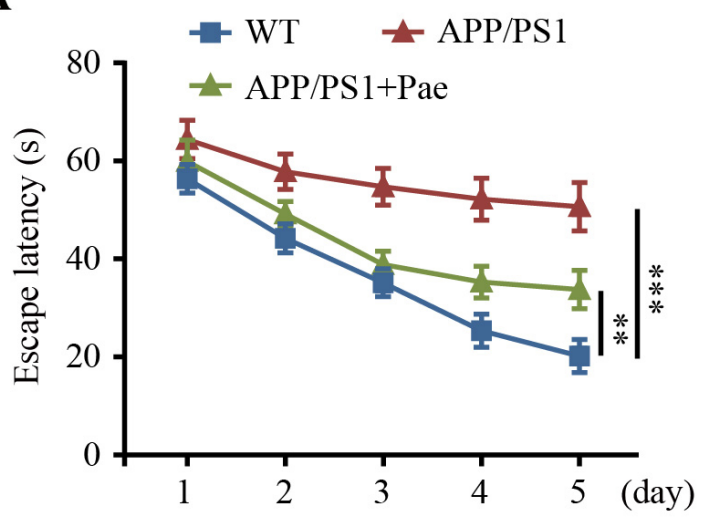

C

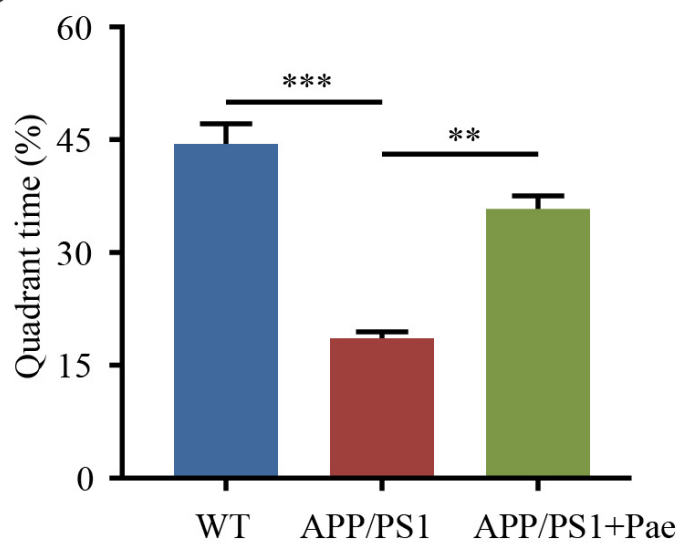

E

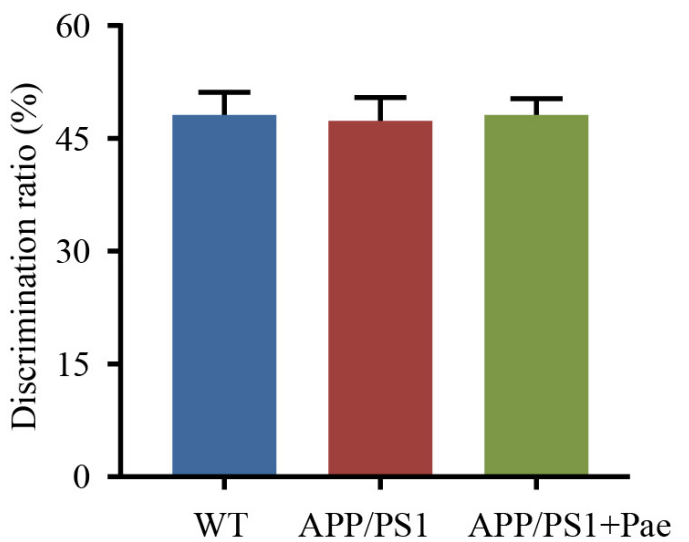

B

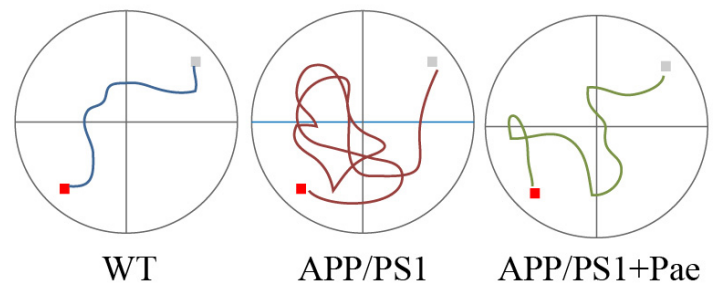

D

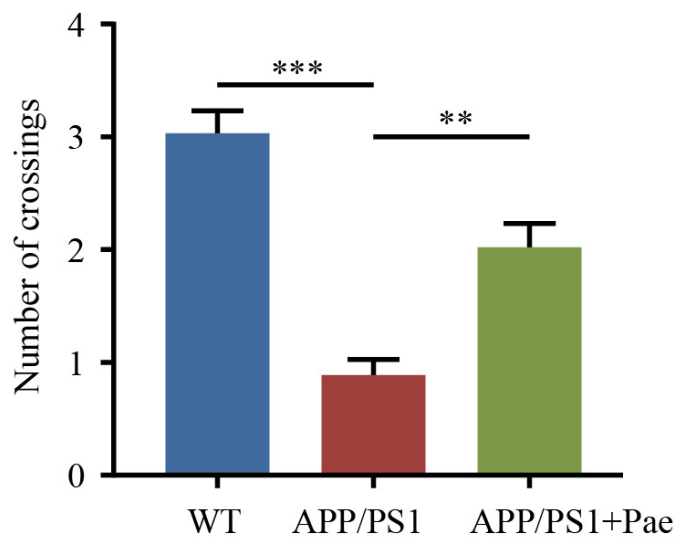

F

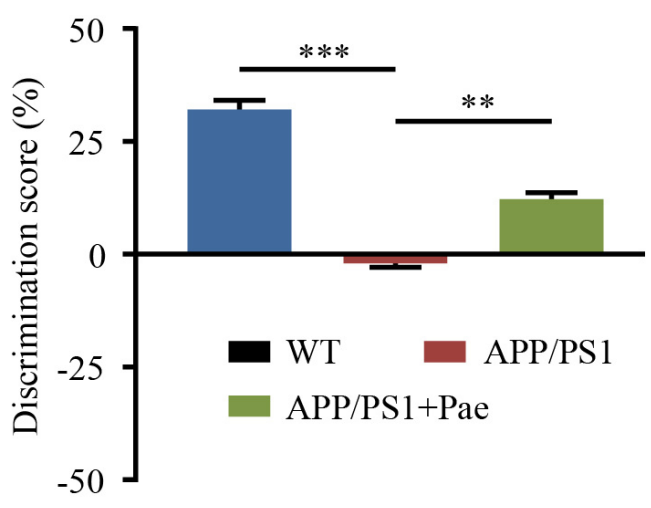

Fig. 1. Paeonol improves neurobehavioral outcomes in APP/PS1 transgenic mice. (A) The time to climb on the hidden platform during the training phase of Morris water maze. (B) Trace map of the mice during the Morris water maze test. (C) The time of exploring hidden platform in the Morris water maze. (D) The number of crossing hidden platform in the Morris water maze. (E) Time of exploring same object in the novel object test. (F) Recognition index performance in the novel object test. Mean \pm SEM was shown $(\mathrm{n}=8) .{ }^{* *} p<0.01,{ }^{* * *} p<0.001$.

\subsection{Western blotting}

Dissected hippocampi were harvested and lysed using RIPA buffer containing protease and phosphatase inhibitors (Beyotime, China). The concentration of protein was measured with a BCA protein assay kit (Pierce, Rockford, USA). Later, equal amounts of protein were separated by SDS-
PAGE and transferred to PVDF membranes (BioRad). After blocking with $5 \%$ skimmed milk for $2 \mathrm{~h}$ at room temperature, they were incubated overnight at $4{ }^{\circ} \mathrm{C}$ with the primary antibody (anti-PSF-95 1:1000, anti-SYN 1:2000, antiMap-2 1:800 or anti- $\beta$-actin 1:1000). On the following day, the membranes were incubated with HRP-conjugated sec- 
A
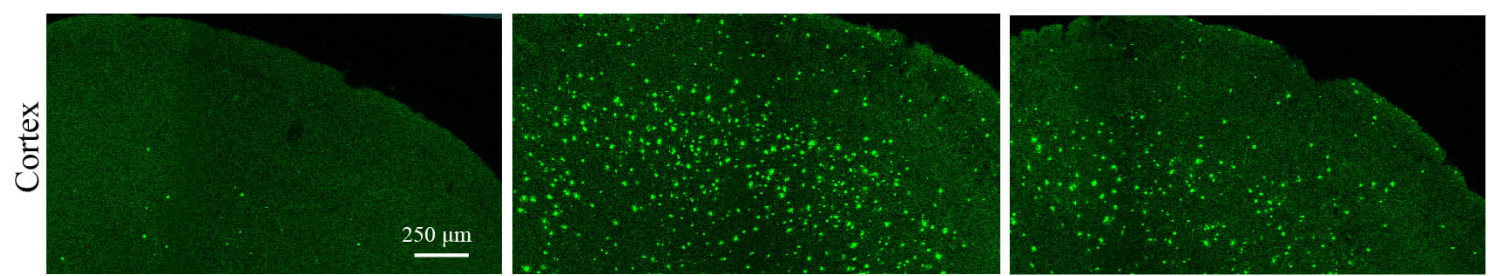

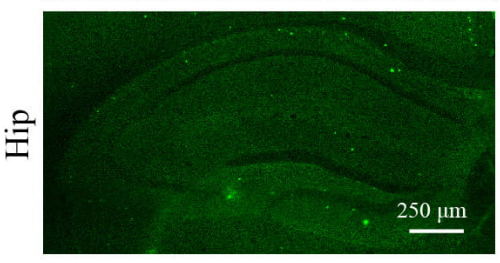

WT

B

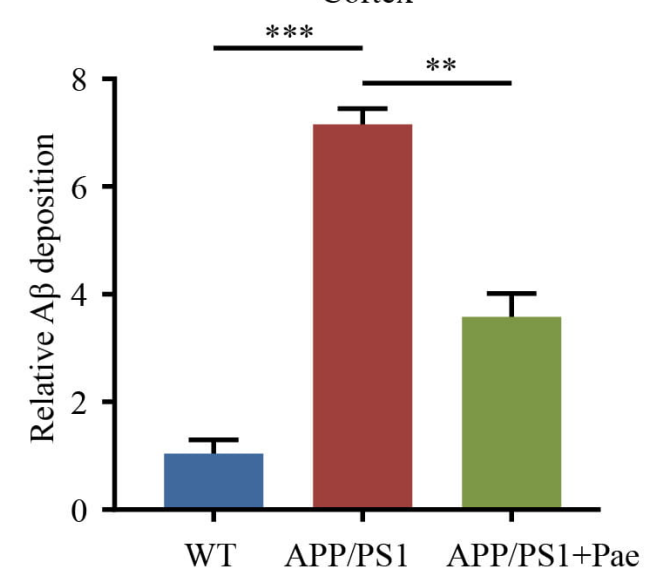

D

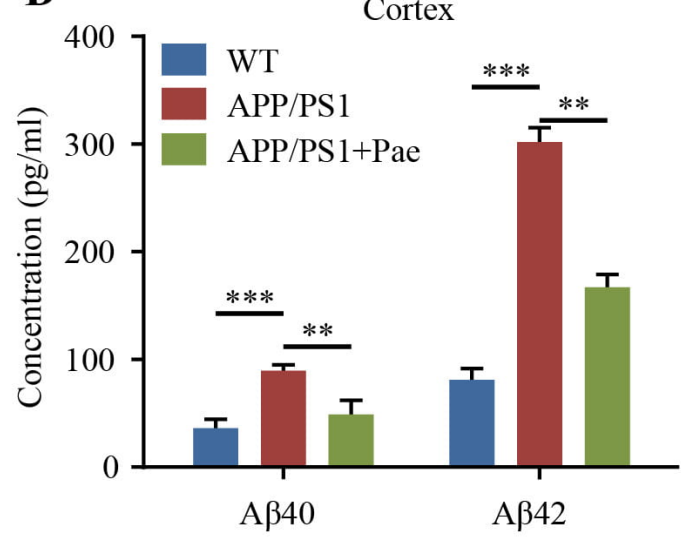

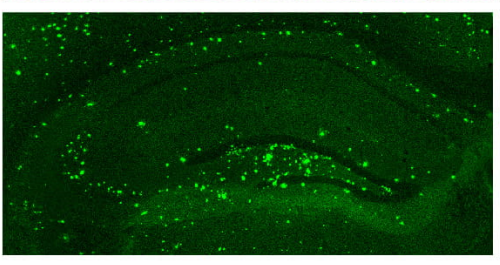

APP/PS1

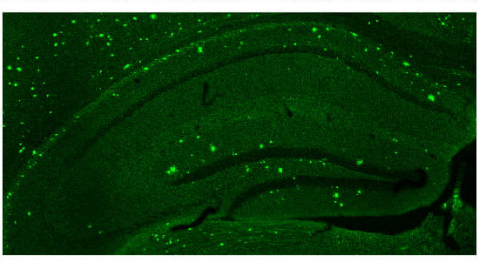

APP/PS1+Pae

C
Hippocampus

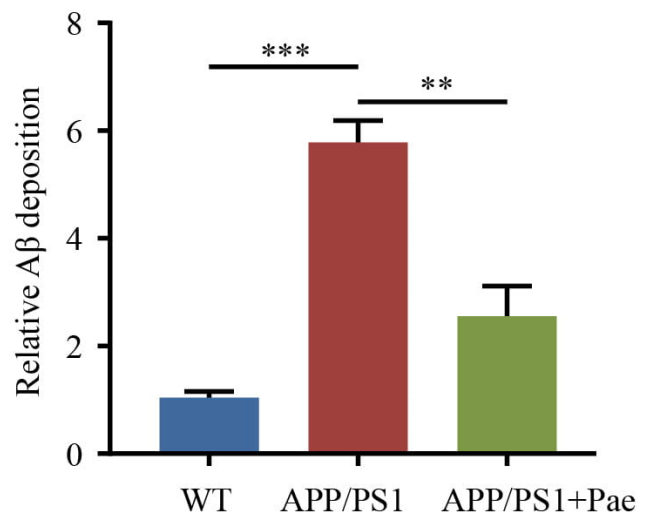

$\mathbf{E}$

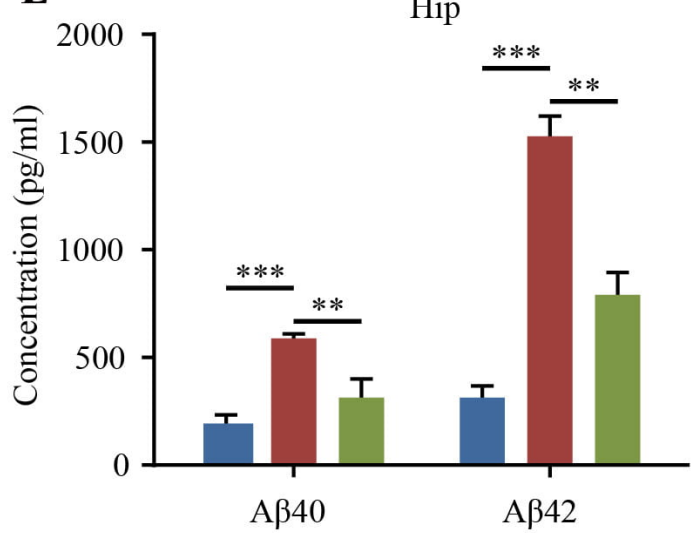

Fig. 2. Paeonol adminstration decreased $\mathbf{A} \beta$ plaques burden in APP/PS1 mice. (A-C) Immunofluorescent labelling and quantification results of $\mathrm{A} \beta$ plaque deposition in the cortex and hippocampus. Scale bar $=200 \mu \mathrm{m}$. (D,E) ELISA results showed a significant reduction in soluble A $\beta 40 / 42$ in both the cortex and hippocampus. Mean \pm SEM was shown $\left(\mathrm{n}=7\right.$ mice). ${ }^{* *} p<0.01{ }^{* * *} p<0.001$.

ondary antibody (1:5000). Each sample was analyzed by immunoblotting at least twice. The positive bands were visualized by enhanced chemiluminescence (Pierce Biotechnology; Sigma-Aldrich Chemical). ImageJ software (National Institutes of Health, Bethesda, MD, USA) was used for the quantification of protein levels.

\subsection{Immunohistochemistry (IHC)}

After being perfused transcardially with ice-cold $0.1 \mathrm{M}$ phosphate-buffered saline (PBS) and post fixed with $4 \%$ paraformaldehyde (PFA) [20], 4- $\mu$ m thick coronal sections were used for Nissl staining [21], and $15-\mu \mathrm{m}$ coronal sections were prepared for immunohistochemistry. Subsequently, 
A

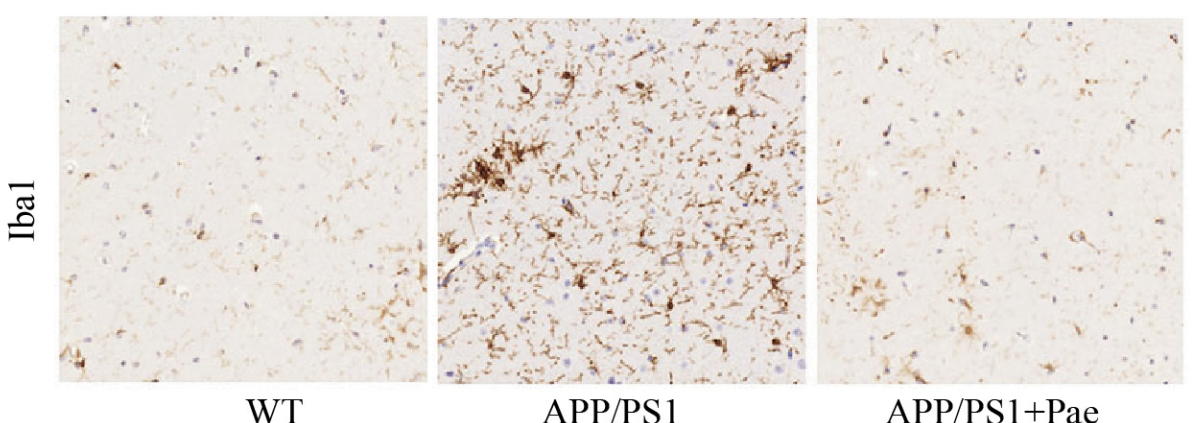

B

Iba1

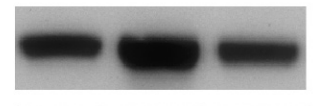

$\beta$-actin

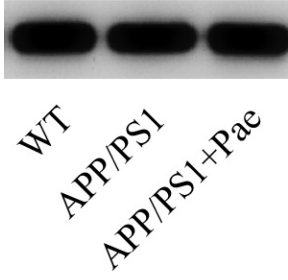

APP/PS1

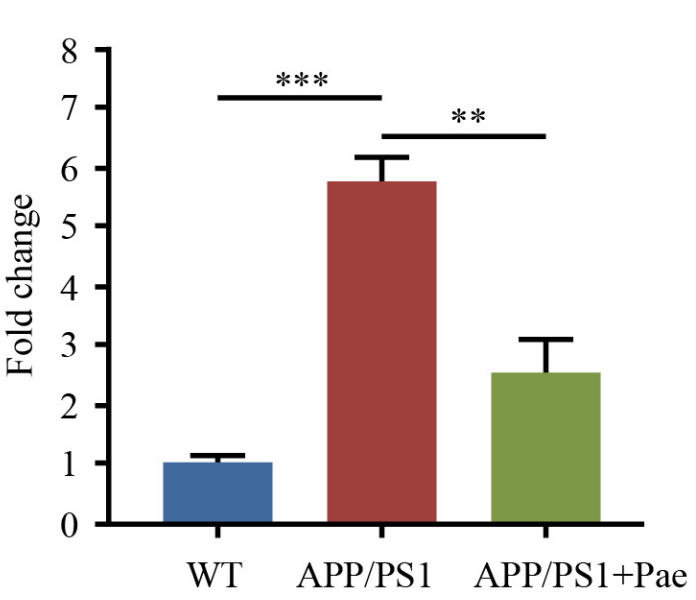

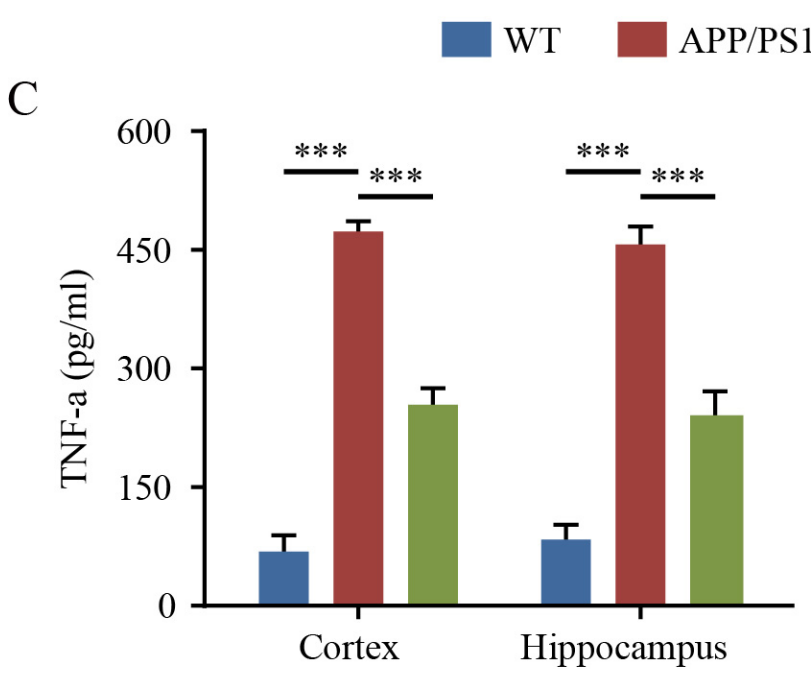

APP/PS1+Pae

$\mathrm{D}$

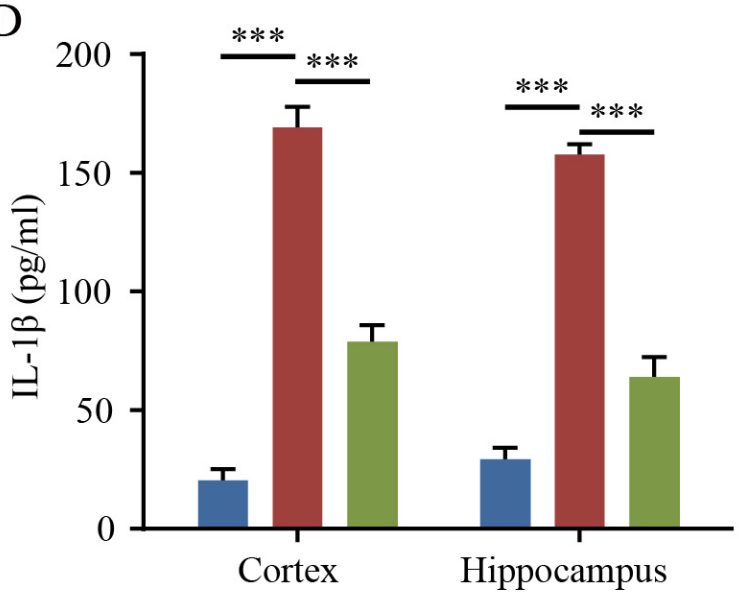

Fig. 3. Paeonol treatment caused microglia inactivation and decreased neuroinflammation in APP/PS1 mice. (A) Immunohistochemistry for microglia markers ionized calcium-binding adapter molecule 1 (Iba1) in the hippocampus. Scale bars, $20 \mu \mathrm{m}$. (B) Western blot band results of Iba1 expression and (E-G) the results of statistical analyses. (C,D) TNF- $\alpha$ and IL-1 $\beta$ levels in the cortex and hippocampus were detected by ELISA. Mean \pm SEM was shown (n=5 mice). ${ }^{* *} p<0.01,{ }^{* * *} p<0.001$.

sections were blocked in $10 \%$ normal goat serum (30 min) and incubated with primary antibodies: anti-Iba-1 (Abcam, Ab178847, 1:400) at $4{ }^{\circ} \mathrm{C}$ overnight. The secondary antibody was allowed to react with the sections. After washing with PBS three times with 0.1 M PBS, the sections were further reacted with $H R P$ and visualized with $D A B$.

\subsection{Statistical analysis}

Data in this study were analyzed using GraphPad Prism 7.0 software (GraphPad Inc., La Jolla, CA, USA) and results are shown as mean \pm SEM. One-way ANOVA or Two-way RM ANOVA was used for the multigroup comparison according to experimental requirements; $p<0.05$ was considered statistically significant. 


\section{Results}

3.1 Paeonol treatment improves cognitive neurobehavioral performance in APP/PS1 mice

Impaired cognitive performance is well observed in the APP/PS1 AD model. After paeonol or vehicle treatment, the behavior of the three groups were tested using the Morris Water Maze (MWM) and novel object recognition (NOR). In the MWM, the results showed that the paeonol treated APP/PS1 mice took less time to reach the hidden platform than the vehicle-treated APP/PS1 mice during the training phase (Fig. 1A,B). The average swimming speed did not differ among the three groups. In the following probe trials, the APP/PS1 mice explored a shorter time, as well as performing fewer crossings into the target quadrant than the WT group. However, both parameters were significantly improved by paeonol treatment (Fig. 1C,D). In the NOR test, as expected, vehicle-APP/PS1 mice could not distinguish the novel from the familiar object, while paeonol-treat APP/PS1 mice displayed a significantly higher discrimination index, with better cognitive performance (Fig. 1E,F).

\subsection{Paeonol administration alleviated $A \beta$ deposition in APP/PS1 mice}

After behavioral testing, the mice were sacrificed, and $\mathrm{A} \beta$ pathology was visualized using immunofluorescence (Fig. 2A). Cortical and hippocampal sections of APP/PS1 mice showed apparent $\mathrm{A} \beta$ plaque deposits (Fig. 2B,C). However, sections of paeonol-treated APP/PS1 mice exhibited reduced positive area of $\mathrm{A} \beta$ plaque (Fig. 2B,C). Soluble $\mathrm{A} \beta 40$ and $\mathrm{A} \beta 42$ concentrations in the cortex and hippocampus were assessed using sandwich ELISAs. As shown in Fig. $2 \mathrm{D}, \mathrm{E}$, the level of soluble $\mathrm{A} \beta 40$ and $\mathrm{A} \beta 42$ were significantly lower within the cortex and hippocampus of the paeonol-treated group than in the vehicle control group. 3.3 Paeonol suppresses the activation of the microglial and
inflammatory response in the hippocampus of APP/PS1 mice

We carried out Iba1 immunofluorescence staining to assess whether paeonol could inhibit microglial activation in APP/PS1 mice. Results showed that the activation of microglia in the hippocampus of paeonol-treated mice were inhibited compared with vehicle-treated APP/PS1 mice (Fig. 3A) and this was further confirmed by immunoblot analysis (Fig. 3B). We also studied the effects of paeonol on the expression of inflammation-related molecules in the cortex and hippocampus of APP/PS1 mice, with levels of TNF$\alpha$ and IL- $1 \beta$ were detected by ELISA. Consistent with the above results, it was clearly evident that the cytokine inflammatory response in the paeonol-treated APP/PS1 mice was less prominent (Fig. 3C,D).

\subsection{Paeonol treatment ameliorated APP/PS1 mice hippocampal neuronal damage}

Previous results prompted us to further evaluate hippocampus neural damage. According to the results of Nissl staining, the decreased number of Nissl-positive cells within CA1 and CA3 hippocampal subregions revealed a signifi- cant protective effect of paeonol (Fig. 4A). It was proved that APP/PS1 mice administered paeonol exhibited a significantly decreased proportion of Nissl-positive cells (Fig. 4B). This provides direct evidence that paeonol confers a neuroprotective effect. APP/PS1 mice have been shown to undergo spine morphology changes and decreases in spine density. To examine whether paeonol treatment achieved a therapeutic effect on synaptic connectivity, hippocampal neuronal spines from each group were GFP-labelled for morphological analysis (Fig. 4C). Spine loss was represented by diminished GFPlabelling, and this was observed in the hippocampus pyramidal neurons of APP/PS1 mice. Paeonol administration reversed this spine density reduction, implying a neuroprotective effect of paeonol on synaptic impairment (Fig. 4D).

\subsection{Paeonol treatment rescued hippocampal synaptic plasticity impairment in APP/PS1 mice}

Long-term potentiation (LTP) was induced within the hippocampus to test the hypothesis that paeonol improves cognitive recovery by rescuing functional synaptic connections in this brain region in APP/PS1 mice. The slope of field excitatory postsynaptic potential (fEPSP) induced by stimulation was calculated in each study group. Paeonol could effectively rescue excitatory synaptic current deficit in APP/PS1 mice after the high-frequency stimulation protocol, as demonstrated in the improved LTP found in paeonoltreated mice (Fig. 5A,B). The expression of molecular markers of synaptic damage was further examined. As shown in Fig. 5C-F, we observed that compared with controls APP/PS1 mice, the levels of post-synaptic protein-95 (PSD95), synaptophysin (SYN), and dendritic-related molecular microtubule-associated protein-2 (MAP-2) were significantly increased in mice receiving paeonol treatment. These molecular expression findings further validate the synaptic protection of paeonol witnessed in functional performance tests.

\section{Discussion}

As the major contributor to dementia, memory and cognitive impairment are the two prominent features of $\mathrm{AD}$ [22]. There is an urgent need to develop novel effective preventative treatments that are accompanied by minimal adverse effects. In this study, we utilized a rodent AD model to assess the potential protective effects of paeonol against AD-like behavioral and pathological manifestations. Our results provide evidence that paeonol exerts a therapeutic effect in APP/PS1 mice. Paeonol treatment for 3 weeks effectively prevented high $\mathrm{A} \beta$ plaque burden, reducing $\mathrm{A} \beta 42, \mathrm{~A} \beta 40$ and neuroinflammation levels in the brains of APP/PS1 mice. Furthermore, paeonol reversed many of the neural morphological and plasticity changes introduced by the APP/PS1 transgenes. These results provide direct evidence of the effect of paeonol on AD.

To attain confirmation of the effects of paeonol on APP/PS1 mice while considering the $3 \mathrm{R}$ principle, only males were tested in this study. In the hippocampus-dependent 
A
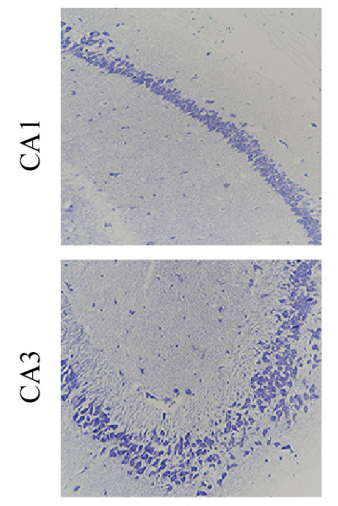

WT

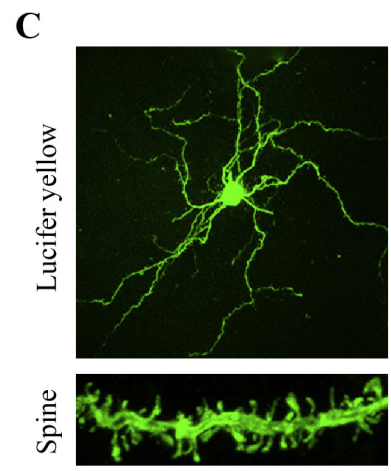

WT
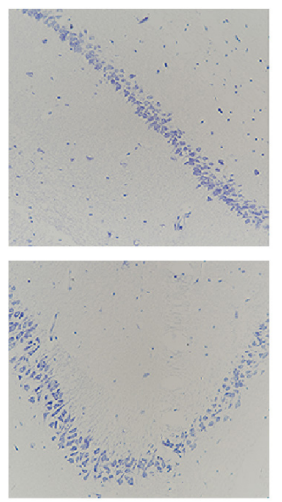

APP/PS1

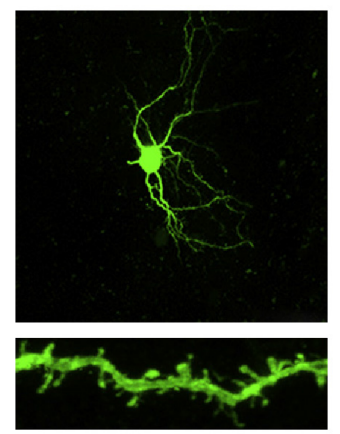

APP/PS1
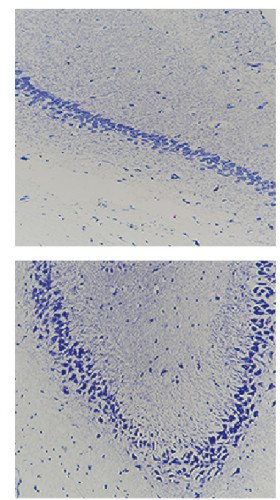

APP/PS1+Pae

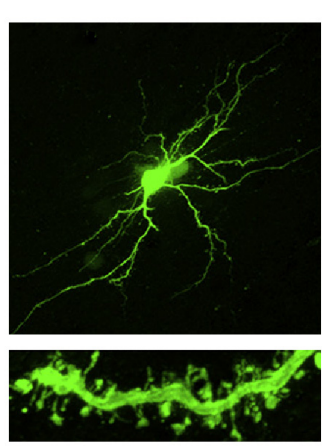

APP/PS1+Pae
B

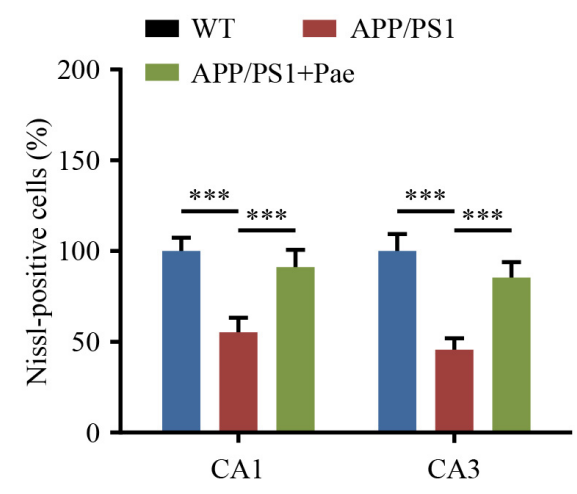

D

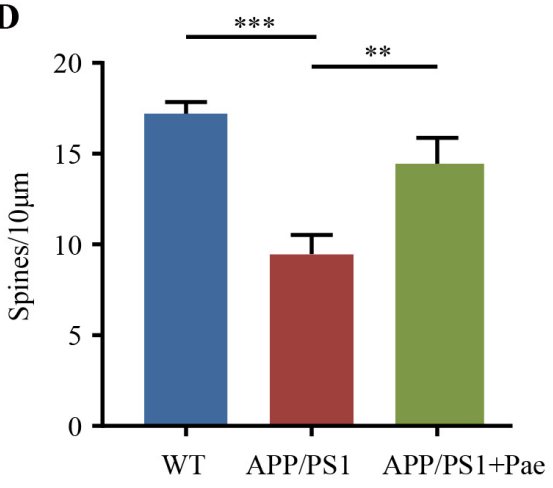

Fig. 4. Paeonol treatment inhibited neuronal damage in the hippocampus of APP/PS1 mice. (A) Nissl staining results of the hippocampal CA1 and CA3 regions. (B) Quantification of Nissl-positive dead cells in the hippocampus of CA1 and CA3 regions. (C) Representative photomicrographs of EGFP-labeling in CA1 neurons under treatment with Paeonol. Scale bar: $10 \mu \mathrm{m}$. (D) Quantification of the density of dendritic spines from randomly selected dendritic segments of neurons. Mean \pm SEM was shown ( $\mathrm{n}=5$ mice). ${ }^{* *} p<0.01,{ }^{* * *} p<0.001$.

memory MWM test, paeonol-treated APP/PS1 mice showed enhanced learning compared to their vehicle-treated counterparts. Indeed, both groups of mice could learn to locate the platform position within several days. However, paeonoltreated APP/PS1 mice showed a faster learning curve, achieving a significant improvement by the end of the learning period. In another hippocampus-dependent cognitional behaviors test, the NOR test, paeonol-treated APP/PS1 mice spent more time investigating the novel object, which further confirmed our hypothesis that paeonol has an effect on AD. This is well in agreement with previous studies' reports that this compound can improve learning behavior in rodent brain disease models [13, 14].

APP overexpression has been shown to be a central mediator of microglia activation in the pathogenesis of AD [23]. Amyloid plaques deposited in the AD brain are closely associated with a local inflammatory process, with neuroinflammation a key factor contributing to the further production of $\mathrm{A} \beta$ and neuronal loss in $\mathrm{AD}$ [4]. It is known that the severity of cognitive impairment is positively correlated with the degree of $\mathrm{A} \beta$ plaque accumulation [2]. In this study, we provide evidence that the alleviation of $\mathrm{A} \beta$ pathology can lead to the amelioration of cognitive behavioral deficits in
APP/PS1 mice. Our study also demonstrated, for the first time, that paeonol can significantly inhibit $\mathrm{A} \beta$ pathological progression in a rodent AD model that simulates the manifestation and pathological processes of $\mathrm{AD}$ in humans. $\mathrm{A} \beta$ has been reported to be proinflammatory and to regulate microglial phenotype in AD [24]. Microglia, together with astrocytes, create a mesh of activity that leads to inflammation and the progression of AD. In our study, we have shown a reduction in hallmarks of microglia activity through immunohistochemistry, which leads us to believe that paeonol has an effect on microglia inhibition in this mouse model of AD. Signaling from microglia through pro-inflammatory cytokines, such as TNF- $\alpha$, can lead to the activation of apoptosis in neurons through caspase-dependent pathways. In the APP/PS1 mice, we observed a decrease in microglia activation combined with a reduction in TNF- $\alpha$ and IL- $1 \beta$ levels in the hippocampus, which represents a series of mechanisms that may protect the APP/PS1 mice from neuronal death. Thus, A $\beta$ reduction by paeonol treatment in APP/PS1 could have contributed to microglial and proinflammatory cytokine downregulation. Earlier in vivo and in vitro results both indicate that paeonol may be a microglia inhibitor $[25,26]$. This inhibitory effect was also demonstrated in our AD mice. 


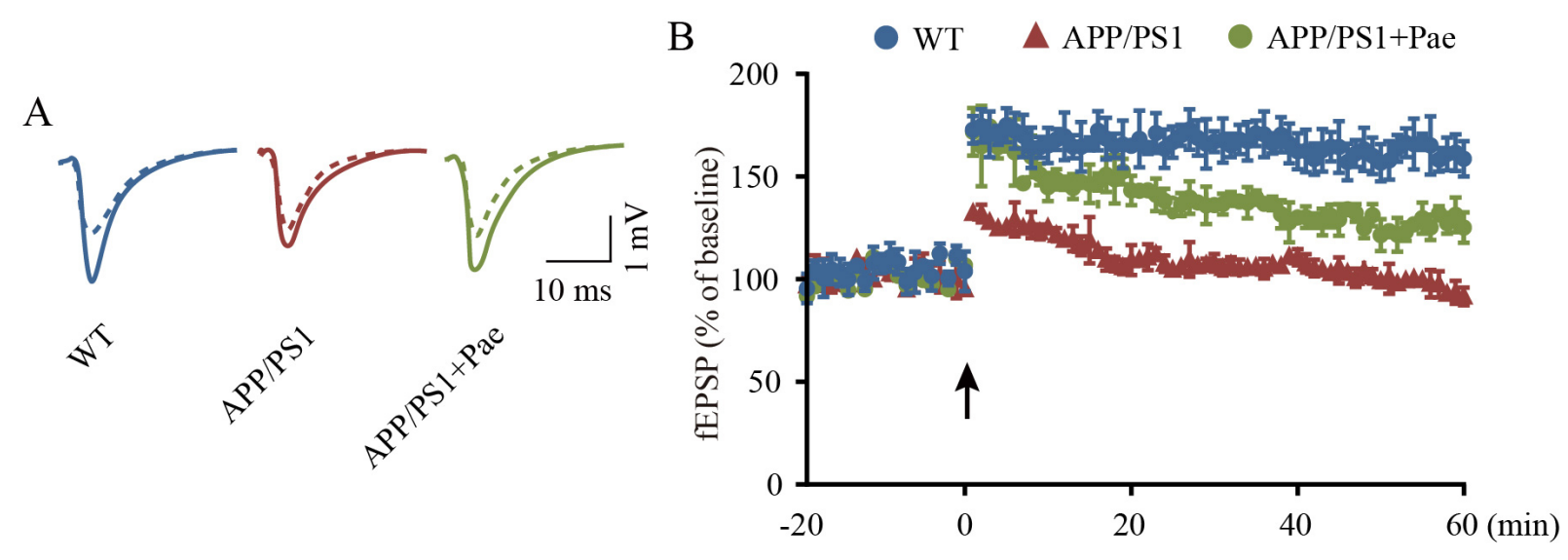

C

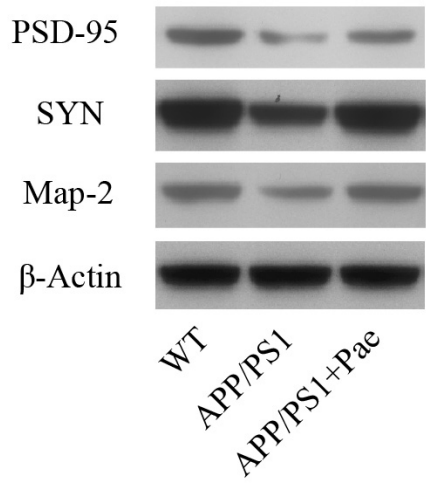

E

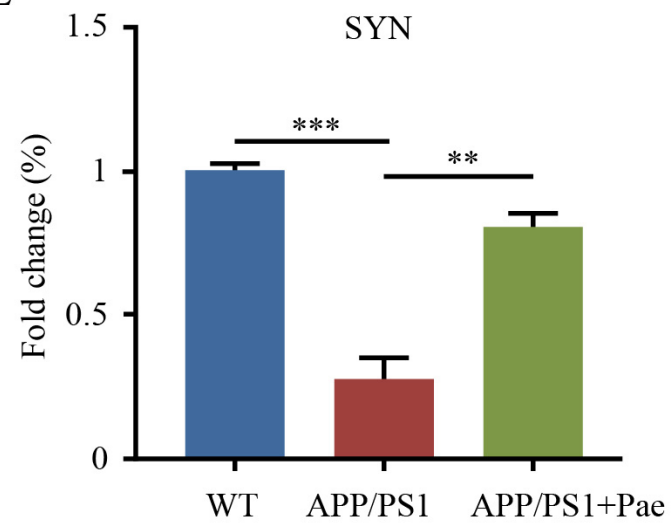

D

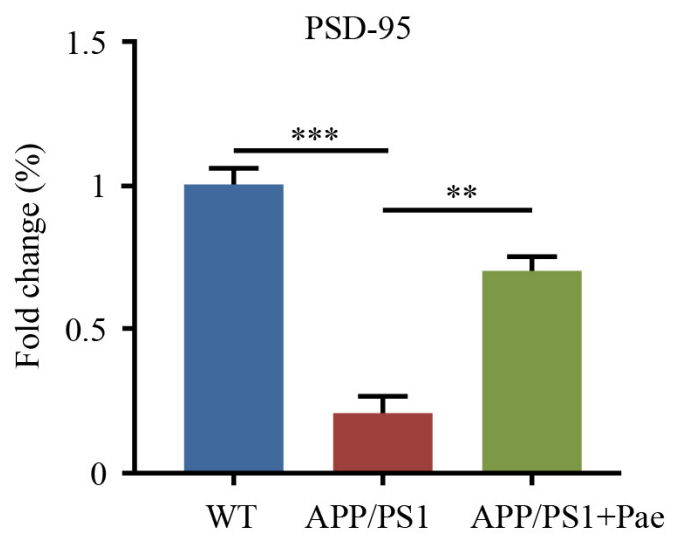

F

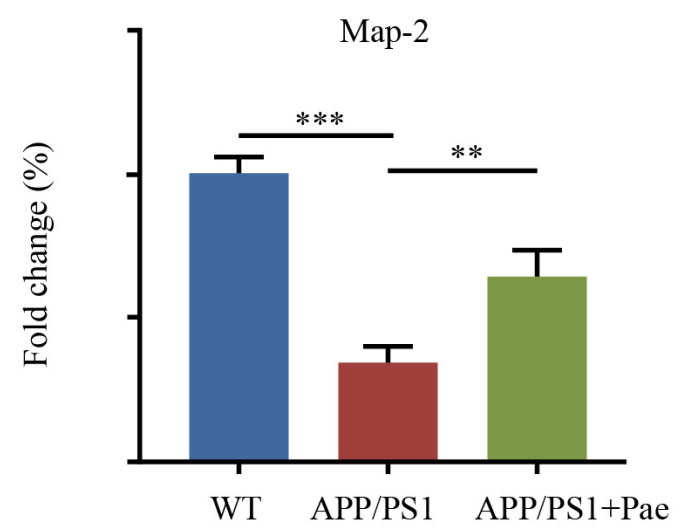

Fig. 5. Paeonol administration promote synaptic plasticity in APP/PS1 mice. (A) Traces of hippocampal LTP induced with high frequency stimulation. (B) Normalized fEPSP slopes of HFS-induced LTP. (C-F) Representative immunoblots and the relative levels of PSD-95, SYP, and MAP-2. Mean \pm SEM was shown ( $\mathrm{n}=5$ mice). ${ }^{* *} p<0.01,{ }^{* * *} p<0.001$.

The hippocampus is one of the main areas of the brain affected by neuronal injury and synaptic plasticity in AD. Previous studies have shown that $\mathrm{A} \beta$ could mediate synapse dysfunction and loss during neurodegeneration [27]. As activated microglia are thought to mediate synaptic stripping, we sought to determine whether paeonol could affect dendritic spine number in APP/PS1 mice. To that end, we treated hippocampus neurons with GFP-tagged AAV and counted den- dritic spines in the CA1 region of the hippocampus. Total spine density was significantly decreased in APP/PS1 compared to WT mice, and a recovery of this effect was observed following paeonol administration. LTP is believed to be a synaptic mechanism underlying the storage of memories in the brain [28]. Therefore, enhancement of LTP by paeonol administration explains the synaptic recovery accompanied by improved behavioral outcomes in APP/PS1 
mice. In line with this idea, several important protein markers associated with synaptic plasticity showed the same trend after paeonol administration, and these trends were accompanied by improved cognitive function. These results indicate that paeonol administration can restore synaptic impairment, in accordance with previous research showing that paeonol could increase the frequency of miniature excitatory postsynaptic currents and spontaneous excitatory postsynaptic currents, thus promoting LTP [29]. Our experimental results further confirm this phenomenon. The paeonol dose adopted was derived from previous study [16]. The choice of the tested dose was based on safety data, and does not correspond to the minimal effective dose. This study could be helpful in the selection of dosages for future AD disease models. We did not perform a dosing study as we were mainly interested in evidencing the potential effectiveness of paeonol. Future experiments will explore minimal and optimal doses and the dose-response relationship of paeonol for AD treatment in mice.

\section{Conclusions}

In summary, we showed that paeonol could reverse cognitive impairment and $\mathrm{A} \beta$-related pathological processes in APP/PS1 mice. Chronic paeonol treatment was effective in exerting neuro- and synaptic plasticity protective effects in APP/PS1 mice. Though the fine pharmacologic targets of paeonol should be determined in future study, our present study adds to the growing body of evidence that paeonol could be a relevant therapy in the clinical management of ADassociated cognitive disorders.

\section{Abbreviations}

$\mathrm{AD}$, Alzheimer's disease; $\mathrm{A} \beta$, amyloid- $\beta$; APP/PS1, APPswe/PSEN1dE9; WT, wild-type; MWM, Morris water maze; NOR, Novel object recognition; Thio S, Thioflavin S; AAV, adeno-associated virus; IHC, Immunohistochemistry; LTP, Long-term potentiation.

\section{Author contributions}

SXM conceived and designed the experiments; BW performed the experiments; WL analyzed the data; WL contributed reagents and materials; SXM wrote the paper.

\section{Ethics approval and consent to participate}

Animals were purchased from Beijing Vital River Laboratory Animal Technology Co., Ltd. (Beijing, China) and raised and handled at the Laboratory Animal Center of the Shanghai Jiao Tong University. All animal experiments were carried out in accordance with the guidelines of the Guidelines for Animal Care and Use of China, and the experimental schemes were approved by the animal ethics committee of Shanghai Jiao Tong University (SYXK-2020-0050).

\section{Acknowledgment}

We thank three anonymous reviewers for excellent criticism of the article.

\section{Funding}

This study was supported by Project of Shanghai Science and Technology Commission (19401970600); Project of Shanghai Science and Technology Commission (19401932500).

\section{Conflict of interest}

The authors declare no conflict of interest.

\section{References}

[1] Li D, Liu C. Hierarchical chemical determination of amyloid polymorphs in neurodegenerative disease. Nature Chemical Biology. 2021; 17: 237-245.

[2] Mecozzi V, Berman D, Simoes S, Vetanovetz C, Awal M, Patel V, et al. Small, Pharmacological chaperones stabilize retromer to limit APP processing. Nature Chemical Biology. 2014; 10: 443-449.

[3] Crews L, Masliah E. Molecular mechanisms of neurodegeneration in Alzheimer's disease. Human Molecular Genetics. 2010; 19: 1220.

[4] Janus C, Pearson J, Mclaurin JA, Mathews PM, Jiang Y, Schmidt $\mathrm{SD}$, et al. A $\beta$ peptide immunization reduces behavioural impairment and plaquesin a model of Alzheimer's disease. Nature. 2000; 408: 979-982.

[5] Su D, Chai Y, Yang J, Wang X, Liu Y, Ma J, et al. LentivirusCarried microRNA-195 Rescues Memory Deficits of Alzheimer's Disease Transgenic Mouse by Attenuating the Generation of Amyloid Plaques. Frontiers in Pharmacology. 2021; 12: 633805.

[6] Liu W, Sun F, Wan M, Jiang F, Bo X, Lin L, et al. $\beta$-Sheet Breaker Peptide-HPYD for the Treatment of Alzheimer's Disease: Primary Studies on Behavioral Test and Transcriptional Profiling. Frontiers in Pharmacology. 2017; 8: 969.

[7] Kuroda E, Nishimura K, Kawanishi S, Sueyoshi M, Ueno F, Toji $\mathrm{Y}$, et al. Mouse Bone Marrow-derived Microglia-like Cells Secrete Transforming Growth Factor- $\beta 1$ and Promote Microglial $\mathrm{A} \beta$ Phagocytosis and Reduction of Brain A $\beta$. Neuroscience. 2020; 438: 217-228.

[8] Cooper NR, Bradt BM, O'Barr S, Yu JX. Focal inflammation in the brain. Immunologic Research. 2000; 21: 159-165.

[9] Cacabelos R, Torrellas C, López-Muñoz F. Epigenomics of Alzheimer's Disease. Journal of Experimental \& Clinical Medicine 2014; 6: 75-82.

[10] Kim S, Park M, Park Y, Na H, Kim H, Shin M, et al. Paeonol inhibits anaphylactic reaction by regulating histamine and TNFalpha. International Immunopharmacology. 2004; 4: 279-287.

[11] He X, Cai Q, Li J, Guo W. Involvement of brain-gut axis in treatment of cerebral infarction by $\beta$-asaron and paeonol. Neuroscience Letters. 2018; 666: 78-84.

[12] Zhu X, Chen J, Han F, Pan C, Zhuang T, Cai Y, et al. Novel antidepressant effects of Paeonol alleviate neuronal injury with concomitant alterations in BDNF, Rac1 and RhoA levels in chronic unpredictable mild stress rats. Psychopharmacology. 2018; 235: 2177-2191.

[13] Jin H, Wang M, Wang J, Cao H, Niu W, Du L. Paeonol attenuates isoflurane anesthesia-induced hippocampal neurotoxicity via modulation of JNK/ERK/P38MAPK pathway and regulates histone acetylation in neonatal rat. Journal of Maternal-Fetal \& Neonatal Medicine. 2020; 33: 81-91.

[14] Han F, Zhuang T, Chen J, Zhu X, Cai Y, Lu Y. Novel derivative of Paeonol, Paeononlsilatie sodium, alleviates behavioral damage and hippocampal dendritic injury in Alzheimer's disease concur- 
rent with cofilin1/phosphorylated-cofilin1 and RAC1/CDC42 alterations in rats. PLoS ONE. 2017; 12: e0185102.

[15] Collins J, King A, Woodhouse A, Kirkcaldie M, Vickers J. The effect of focal brain injury on beta-amyloid plaque deposition, inflammation and synapses in the APP/PS1 mouse model of Alzheimer's disease. Experimental Neurology. 2015; 267: 219229.

[16] Shi X, Chen Y, Liu H, Qu H. Therapeutic effects of paeonol on methyl-4-phenyl-1,2,3,6-tetrahydropyridine/probenecidinduced Parkinson's disease in mice. Molecular Medicine Reports. 2016; 14: 2397-2404.

[17] Torrisi S, Geraci F, Tropea M, Grasso M, Caruso G, Fidilio A, et al. Fluoxetine and Vortioxetine Reverse Depressive-Like Phenotype and Memory Deficits Induced by $\mathrm{A} \beta$ Oligomers in Mice: A Key Role of Transforming Growth Factor- $\beta 1$. Frontiers in Pharmacology. 2019; 10: 693.

[18] Long L, Yu Z, Chen S, Wu J, Liu Y, Peng J, et al. Pretreatment of Huoxue Jiedu Formula Ameliorates Myocardial Ischaemia/Reperfusion Injury by Decreasing Autophagy via Activation of the PI3K/AKT/mTOR Pathway. Frontiers in Pharmacology. 2021; 12: 608790.

[19] Ernst R, Krogager T, Maywood E, Zanchi R, Beránek V, Elliott T, et al. Genetic code expansion in the mouse brain. Nature Chemical Biology. 2016; 12: 776-778.

[20] Yang Y, Li S, Huang H, Lv J, Chen S, Pires Dias A, et al. Comparison of the Protective Effects of Ginsenosides Rb1 and Rg1 on Improving Cognitive Deficits in SAMP8 Mice Based on Anti-Neuroinflammation Mechanism. Frontiers in Pharmacology. 2020; 11: 834.

[21] Kupats E, Stelfa G, Zvejniece B, Grinberga S, Vavers E, MakreckaKuka M, et al. Mitochondrial-Protective Effects of R-Phenibut af- ter Experimental Traumatic Brain Injury. Oxidative Medicine and Cellular Longevity. 2020; 2020: 9364598.

[22] Selkoe DJ. The molecular pathology of Alzheimer's disease. Neuron. 2012; 22: 11-22.

[23] Francis YI, Fà M, Ashraf H, Zhang H, Staniszewski S, Latchman DS, et al. Dysregulation of histone acetylation in the APP/PS1 mouse model of Alzheimer's disease. Journal of Alzheimers Disease. 2009; 18: 131-139.

[24] Wang H, Zhang T, Huang J, Xiang J, Chen J, Fu J, et al. Edaravone Attenuates the Proinflammatory Response in Amyloid- $\beta$-Treated Microglia by Inhibiting NLRP3 Inflammasome-Mediated IL-1 $\beta$ Secretion. Cellular Physiology and Biochemistry. 2017; 43: $1113-$ 1125.

[25] He L, Tong X, Zeng J, Tu Y, Wu S, Li M, et al. Paeonol Suppresses Neuroinflammatory Responses in LPS-Activated Microglia Cells. Inflammation. 2016; 39: 1904-1917.

[26] Jung E, Hwang J, Kwon M, Kim K, Cho H, Lyoo I, et al. A tryptamine-paeonol hybridization compound inhibits LPSmediated inflammation in BV2 cells. Neurochemistry International. 2016; 100: 35-43.

[27] Dore K, Carrico Z, Alfonso S, Marino M, Koymans K, Kessels H, et al. PSD-95 protects synapses from $\beta$-amyloid. Cell Reports 2021 ; 35: 109194

[28] Bouteiller J, Baudry M, Allam S, Greget R, Bischoff S, Berger T. Modeling glutamatergic synapses: insights into mechanisms regulating synaptic efficacy. Journal of Integrative Neuroscience. 2008; 7: 85-197.

[29] Yang C, Lu G, Hsu S, MacDonald I, Chiou L, Hung S, et al. Paeonol promotes hippocampal synaptic transmission: The role of the Kv2.1 potassium channel. European Journal of Pharmacology. 2018; 827: 227-237. 